Удк 316.02

\title{
НЕЙРОНАУЧНЫЕ ИНСТРУМЕНТЫ В СОВРЕМЕННОМ СОЦИОЛОГИЧЕСКОМ ПОЗНАНИИ
}

\author{
Смирнова Алина Вадимовна, \\ sazalina@yandex.ru \\ Финансовый университет при Правительстве РФ, \\ Россия, г. Москва, Ленинградский пр., 49
}

Смирнова Алина Вадимовна, аспирантка факультета социологии и массовых коммуникаций Финансового университета при Правительстве РФ.

В статье исследуется относительно новое для науки явление - использование нейронаучного инструментария в разнообразных научных дисциплинах (нейроэкономике, культурной нейронауке, нейропедагогике и др.). Приводится пример проектов и инициатив, имеющих место в настоящее время. Особое внимание уделено перспективе применения подобных методов в рамках изучения социально-поведенческих аспектов, в том числе в пределах организационного устройства. Отмечены подходы к пониманию социальной нейронауки и нейросоциологии. Высказаны опасения социологов, которые становятся преградой для активного внедрения прогрессивных методов в традищионную научную область, описан такой феномен, как «биофобия». Рассмотрены публикации, направленные на изучение нейронаучных методов в практической плоскости: отмечены инновационные идеи, которые могут быть реализованными в области работы с персоналом благодаря достижениям нейроменеджмента и нейролидерства. Представлены выводы исследований нейрофизиологических мотивов принятия решений в различных условиях. Отмечены ключевые возражения, которые обычно возникают в отношении нейронаучного инструментария, используемого в рамках изучения поведенческих аспектов. Продемонстрирована краткая характеристика эксперимента, в рамках которого были изучены процессы, описывающие активность головного мозга лидеров ряда профессиональных сфер, также приведены выводы о корреляции соответствующих процессов с практической ролью руководителей. Дается краткий обзор материалов научно-практической конференции, проведенной на тему нейро прожект менеджмента - научной области, направленной на исследование организационных процессов, в которых особую роль играет команда.

Ключевые слова: Нейронаука, нейроменеджмент, нейролидерство, организационное поведение, социально-поведенческие аспекты, управление персоналом.

В современном мире возможности исследовательского поля расширяются поразительно быстрыми темпами, причиной чему является глобализация, объединяющая умы и ресурсы практически всего мира, включающая активное развитие коммуникационных и цифровых технологий. Однако XXI в., уже в первой четверти показавший свои особенности, существенно отличается от XX в. по тем методам и инструментам, которые становятся катализаторами прогресса: если в прошлом столетии основными двигателями выступали именно информационные новшества, открывшие человечеству совершенно новый мир, отличный от индустриального общественного строя, то на текущий момент ведущей силой перемен можно назвать комплекс когнитивных научных дисциплин, а именно нейронауку [1]. Методы данной области знаний распространяются на широкий спектр научных направлений, который непрерывно расширяется. Цель статьи заключается в более подробном рассмотрении роли нейронаучных исследований в направлении социально-поведенческих аспектов, в том числе в рамках организационного поведения как прикладной отрасли знаний. 
Вопрос использования инструментов нейронауки в области изучения социальных аспектов не слишком широко освящается в научной литературе, вероятно, ввиду относительно молодого возраста направления, недостаточного количества экспериментов и исследований для проведения основательного анализа, а также иных причин, внимание которым будет уделено ниже. Все же в последние годы в зарубежных [2-4] и отечественных [5-7] изданиях можно встретить исследования в области указанной темы. Кроме того, имеют место проекты и инициативы исследования мозга, такие как The BRAIN (Brain Research through Advancing Innovative Neurotechnologies) Initiative, The Human Brain Project, The Human Connectome Project, The Brain/MINDS (Brain Mapping by Integrated Neurotechnologies for Disease Studies), The Brain Dialogue, The China Brain Project, The Korea Brain Initiative [8]. В рамках указанных проектов проводятся разнообразные исследования, направленные на углубление знаний в сфере связей, которые можно проследить между мозговой активностью и человеческим поведением. Речь идет о таких инициативах, как изучение феномена «фантомных толчков», ощущаемых матерью после рождения ребенка; предвосхищение сложности протекания шизофрении; доказательство тесной взаимосвязи внимания и процесса принятия решений; объяснение галлюцинаций у пожилых людей со слабым зрением, порожденных реакцией мозга на периферийные изображения и многих других [9]. Учитывая наличие ряда проектов в данном направлении, доказывающих интерес к нейронаучным исследованиям, а также междисциплинарный характер разработок, который может привести к получению значительных результатов в различных направлениях, стоит отметить перспективность указанной области развития науки, в том числе для использования в широкой области социологического поля и, в частности, исследования поведенческих аспектов в организационных пределах.

Как было отмечено, разнообразие научных областей, так или иначе привлекающих инструменты когнитивных дисциплин, становится все более заметным: вслед за нейроэкономикой, культурной нейронаукой, социальной нейронаукой зарождаются более узкоспециализированные направления - нейроэтика, нейростетика, нейрополитология, нейропедагогика, нейроэпистемология и др. [10]. В контексте рассмотрения социальных аспектов поведения человека и его связи с нейронаучным инструментарием стоит обратить особое внимание на такие области знаний, в которых нейронаучные разработки уже так или иначе показали себя или находятся в процессе закрепления на постоянной основе. К таким отраслям следует отнести в первую очередь социальную нейронауку и нейросоциологию. Следует отметить, что четкого разделения между двумя дефинициями нет. Так, Ю.С. Шкурко четко позиционирует социальную нейронауку как уже частично сформировавшуюся и находящуюся в процессе развития область знаний, изучающую социальное поведение, познание и восприятие, саморегуляцию и развитие индивида, процесс принятия решений, а также нейронные механизмы, которые оказывают влияние на их конструирование [11]. Автор также отмечает не только междисциплинарный характер данной отрасли, но и ее межуровневую функциональность, подразумевающую анализ и систематизацию определенного пласта феноменов с помощью поиска связей и их изучения на трех уровнях: нейронном, когнитивном и социальном. Данный аспект особенно важен для понимания структуры этой научной области: ее исследования направлены, по сути, на тот же предмет, что и у традиционной социологии, однако с привлечением иных механизмов, включающих когнитивные человеческие ресурсы и нейронную активность. Если говорить о нейросоциологии, то в контексте своих работ Ю.С. Шкурко отмечает неоднозначность ее предмета, предполагая, что основное внимание данная область должна уделять нейробиологическим механизмам 
общественных интеракций. Стоит также отметить и существование другого мнения, согласно которому социальная нейронаука представляет собой то же самое, что и нейросоциология [12].

В рамках данной статьи мы не будем подробно останавливаться на определении единственно верной научной области для предмета исследования, поскольку как первое, так и второе направления могли бы стать основой для проведения релевантного анализа, но акцентируем внимание на инструментах - нейронаучных исследованиях.

При всей привлекательности нового инструментария как для науки в целом, так и социально-гуманитарных наук в частности, пока нет оснований говорить о действительно активном использовании методов нейронауки в области социологии. Главной тому причиной, по мнению специалистов, изучающих специфику направления, становится «биофобия» [13]. Есть ряд предубеждений и опасений со стороны социологов, которые удаляют их от регулярного применения нейронаучных технологий. Среди них можно отметить направленность социологических исследований на поведение человека, сформированного в контексте общества, не затрагивающего биологических установок. Ученые порой не могут сочетать социологические и биологические факторы, отдавая предпочтение первым, оттого и характер полученных данных лишен какой-либо перспективы опираться на физиологические моменты в будущем.

Кроме того, ряд исследований нейронаучной сферы привел к результатам, которые не всегда с легкостью будут приняты и одобрены традиционной наукой. В качестве примера можно привести теорию о том, что человек реагирует на уровне мозговой активности на происходящие с ним социальные действия так же, как и на физические. В данном контексте широко известная «пирамида» А. Маслоу теряет актуальность, потому как человек может иметь на физиологические потребности ту же реакцию, как и на потребность в общении или признании [14].

Помимо этого, следует отметить, что, по мнению ряда ученых, несмотря на прогрессивность нейронаучных исследований, пока нет возможности предоставить существенные и обоснованные корреляции между данными, описывающими мозг, и человеческим поведением [15]. На их взгляд, в процессе разъяснения нейронаучных данных, полученных в ходе эксперимента, могут возникнуть неточности в плане соотношения их с социологическим профилем и внедрения в действительность социологического анализа. Тем не менее, важно понимать, что, как биологическое начало, так и социальное развитие не противоречат друг другу, а, наоборот, предоставляют колоссальную возможность изучения человеческого существа с новой точки зрения.

Отличный пример рационального отношения к нейронаучным инструментам отражён в произведении Р. Бертона «Разум VS мозг» [16]. Автор формулирует выводы, к примеру, об изменениях в архитектуре мозга в рамках процесса социализации, о переоценке человеком своих способностей как следствии недостатка когнитивных навыков и знаний. Самый важный итог научного исследования Р. Бертона заключается в том, что он призывает социологов не обобщать данные нейронаучных исследований для формулировки неких закономерностей, а, используя полученные знания, более тщательно изучать мотивы действия человека в рамках социума, определять инструменты, противодействующие наступлению или способствующие исправлению отрицательных социальных последствий.

Помимо вышеназванных дисциплин, стоит отметит еще две относительно молодые научные отрасли, предмет исследований которых также направлен на социальные составляющие, но в более узких рамках, а именно в пределах организационного поведения - речь идет о нейроменеджменте и нейролидерстве. Считается, что название 
«нейроменеджмент» было впервые использовано в начале XXI в. К. Ма [17]. Данная область знаний вызывает интерес ввиду связи с прикладной отраслью деятельности, т. е. выводы, полученные в результате соответствующих исследований, могут быть полезными для управленческого процесса. В зарубежной литературе встречаются весьма интересные публикации, в том числе основанные на анализе поведенческих особенностей, коррелирующих с процессом принятия решений, с точки зрения нейрофизиологии. В одной из подобных статей [18] дается обоснование пристальному вниманию ученых к данной области исследований: в процессе принятия решений, основанных на моральных нормах, человек выступает не только «эмоциональным», но и «рациональным» агентом, стремящимся достигнуть материального результата и изменить социальное положение. Так, авторы ведут речь о функциональном методе спектроскопии в ближней инфракрасной области (fNIRS), с помощью которого есть возможность учитывать уровни концентрации оксигенированного $(\mathrm{O} 2 \mathrm{Hb})$ и деоксигенированного $(\mathrm{HHb})$ гемоглобина в различных жизненных условиях - в профессиональном контексте, в процессе социализации, а также в вариативном нравственном поле (с точки зрения принятия справедливых, несправедливых или нейтральных решений). Во время проведения эксперимента было зафиксировано, что существует различие между активностью оксигенированного $(\mathrm{O} 2 \mathrm{Hb})$ и деоксигенированного $(\mathrm{HHb})$ характера в разных участках мозга в зависимости от психологического состояния человека. К примеру, повышение уровня оксигемоглобина и снижение деоксигемоглобина наблюдаются во вентромедиальной префронтальной коре и дорсолатеральной префронтальной коре, когда человек имеет дело со справедливыми моральными поступками, связанными с профессиональной деятельностью, и в верхней височной доле - в случае наличия несправедливости в условиях социального положения. Кроме того, было выявлено, что увеличивается время реакции человека в моменты возникновения справедливых и несправедливых ситуаций в рамках организационной структуры и социальных интеракций. В итоге авторы пришли к четырем обобщенным выводам: 1) оценочное отношение к выгодам посторонних людей, полученным благодаря принятию того или иного решения, варьируется в зависимости от контекста принятия решений; 2) «стоимость» умственного напряжения в процессе принятия нравственного решения изменяется, основываясь на степени личной вовлеченности в процесс; 3) разнообразные активности частей мозга, вовлеченных в умственные или эмоциональные процессы, становятся ответом на определенный тип нравственной обстановки; 4) справедливость играет особую роль в данном процессе, т. к. является фактором, имеющим возможность осуществлять выбор, основываясь на значительном влиянии как когнитивного, так и нейрофизиологического уровней.

Таким образом, данное исследование показывает корреляцию между поведенческими и нейрофизиологическими процессами в процессе принятия решений, что может быть весьма полезным для дальнейшего изучения процесса принятия решения. Стоит отметить, что сами авторы статьи не претендуют на повсеместную практическую применимость результатов экспериментов, поскольку существует ряд ограничений (например, в данный момент отсутствующий мультиметодологический подход, который позволяет учитывать результаты не только со стороны мозговой активности, имеющие отношение к процессу принятия решений, но и с позиции вегетативной системы, которая непосредственно имеет отношение к эмоциональным компонентам поведения), не позволяющих текущим выводам быть исчерпывающими. Тем не менее подобный итог говорит лишь о том, что полученные результаты и основанные на них предположения следует развивать и двигаться дальше в сторону инновационных прорывов. 
Стоит привести в качестве примера еще одну зарубежную статью, в которой, в том числе, автор ставит в один ряд социальную нейронауку и нейроменеджмент, утверждая, что данные дисциплины, будучи весьма прогрессивными в области исследовательского поля, могут сгенерировать новые знания, используемые лидерами организаций для лучшего координирования командой и повышения эффективности деятельности [19]. В статье обращается внимание на тот факт, что именно инновационные реформы в области экономики и управления могут стать средством решения разноуровневых организационных сложностей, благодаря новому подходу, включающему модернизированный, гибкий, адаптивный менеджмент. Новый вид управления спроектирован для определения того, как функционирует человеческий мозг и как конструируется его поведение, а также для упрощения пути реализации максимального потенциала персонала. Кроме того, авторы уделяют внимание и нейролидерству как относительно новому научному направлению, опирающемуся на три постулата: как лидеры принимают решения и справляются с проблемами; как они управляют своими эмоциями и как взаимодействуют с другими; и способствуют ли они переменам. Наиболее изучаемыми областями нейролидерства являются процесс принятия решений, доверие, самоконтроль, социальные отношения, сотрудничество, влияние, стратегическое мышление, организационное поведение. В данном контексте крайне важно понимать, что и рациональное мышление, и эмоции - неотъемлемая часть процесса принятия решений, однако для того, чтобы проследить подобную связь, необходимо осознавать, как функционирует мозг и, соответственно, весь наш организм.

Говоря об изучении нейроменеджмента в области отечественной научной действительности, стоит упомянуть особую разновидность данной отрасли знаний, а именно нейро прожект менеджмент. Широко освящались вопросы указанного направления на научно-практической конференции в марте 2020 г. [20]. В ее рамках речь шла о проектном менеджменте как особой отрасли знаний, играющей все большую роль в исследованиях, а также нейронаучных инструментах. Особый интерес был уделен команде как основополагающей части прожект менеджмента. Среди высказанных идей и результатов исследований были обозначены следующие: важность нейропсихологических принципов формирования проектной команды, современные тренды применения искусственных нейронных сетей (ИНС) в промышленности, роль нейроменеджмента в построении эффективного взаимодействия руководителя с подчиненными и раскрытии потенциала сотрудника, инструменты нейролидерства как способ мотивировать персонал, избегая сложностей и угроз, и многие другие.

Отметим выводы, касающиеся нейробиологических инструментов в изучении организационного поведения, сформулированные Н.М. Ашканаси, У.Дж. Бэкером и Д.А. Уолдмэном [21]. Они говорят о четырех основных возражениях, которые существуют в отношении нейронаучных методик в рамках исследований поведенческих установок в организационных условиях. Во-первых, речь идет об упрощении или, иными словами, сведении человеческого поведения исключительно к уровням активности и бездействия определенных участков мозга. Авторы указывают на тот факт, что нейронаука должна осознавать всю сложность феномена человеческого взаимодействия, которое формируется как на основе нейронных процессов, так и социальных интеракций. Во-вторых, авторы уделяют внимание технологическому и методологическим аспектам как возможным для появления противоречий. Основные сомнения вызывает метод МРТ, применяемый в подобных экспериментах, однако здесь стоит отметить, что его нельзя списать со счетов для использования в области организационных исследований, однако не нужно забывать о корректном интерпретировании его результатов. Так, по 
мнению авторов, даже незначительные итоги исследований могут стать научно полезными в ином контексте. В-третьих, отмечают недоверие к нейронаучным исследованиям в целом, авторы называют данный пункт возражений «So What?» (англ. - «И что?»). Авторы категорически не согласны с данной позицией и приводят примеры исследований, которые привели к полезным результатам. Одно из них, проведенное с использованием цифровой электроэнцефалограммы, помогло выяснить, что групповые лидеры проявляют большую вовлеченность, по сравнению с другими сотрудниками, в процессе командного обсуждения. Кроме того, приводится пример особого способа тренировки лидеров, основанный на видеоиграх. В итоге, есть возможность натренировать мозг испытуемого использовать желаемые образцы поведения, из-за чего определенная совокупность нейронных связей становится постоянной. Подобный эксперимент становится исполнимым только ввиду готовности тренируемого участвовать в процессе, т. е. наличии у него мотивации взаимодействовать с тренером в виде компьютера. И, наконец, вчетвертых, это зачастую отрицательная реакция, сопровождающая многие открытия в области нейронаучных изысканий, касающихся организационного поведения, как было, к примеру, с понятием эмоционального интеллекта. Важно отметить, что авторы придерживаются мнения о важности эмоционального интеллекта, считая его индивидуальной особенностью, наряду с характером и IQ, влияющими на формирование социальнопсихологических аспектов в рамках организации, в том числе лидерских качеств. Так, нейронаучная методология помогает продвигать идеи существования различного уровня восприятия, подтверждая постулаты практическими исследованиями.

Обратимся к исследованию, проведенному на основе цифровой электроэнцефалограммы Д. Уолдманом, П. Балтазаром и С. Петерсоном [22]. В рамках эксперимента ученые изучали мозговую активность 50 лидеров из разных областей - науки, бизнеса, политики и других. В основе исследования было заложено два вопроса, на которые испытуемым необходимо было сформулировать ответы. В процессе размышлений экспериментаторами фиксировалась мозговая активность респондентов, а именно согласованность между координацией разных частей мозга. Первый - «Могли бы вы описать ваши текущие планы развития организации и планы на ее развитие в будущем?». С помощью данного вопроса была протестирована гипотеза о том, что у людей, обладающих хорошими навыками коммуникации и выстраивания социальных связей, активно работает лобная доля правого полушария головного мозга - проявляется высокая согласованность. Второй - «Заглядывая в будущее, как бы вы сформулировали миссию вашей организации?». Здесь респонденты были разделены на три категории: первая те, кто в ответах отдавал предпочтение местоимению «я», ассоциируя бизнес компании с собственной персоной; третья - те, кто видел организацию как единое целое, используя местоимение «мы», где он сам - лишь элемент системы; вторая - промежуточная позиция между первой и третьей категориями. В итоге ученые получили следующие результаты. Как и предсказывалось, активность лобной доли правого полушария была отмечена за теми лидерами, кто проявил себя как сфокусированный на социальном видении. Так, один из респондентов, употребляющий при ответе на второй вопрос местоимение «мы», показавший себя как социально ответственный сотрудник, признанный коллегами вдохновляющим/харизматичным лидером, продемонстрировал результат согласованности около $69 \%$. В отличие от другого лидера, чей показатель согласованности составил $17 \%$. Стоит отметить, что второй респондент, который оказался руководителем, в чьей компании высокая текучка кадров, при его стремительном карьерном росте, получил один из самых низших баллов в рейтинге вдохновляющих/харизматичных лидеров по оценке коллег. 
Итак, несмотря на сложившееся представление о нейронаучной методологии как порой противоречащей исконному социологическому подходу, нельзя не обратить внимание на то, что результаты, полученные в результате нейронаучных изысканий, не могут оставаться незамеченными в силу их новизны и зачастую передовых открытий. Ввиду вышесказанного социологам крайне важно дать возможность новым областям науки, не противоречащим традиционным, а лишь дополняющим и уточняющим их, внедряться в систему общественного познания. Помимо этого, нейронаучные инновации помогают совершенствовать и прикладное управленческое поле с помощью изучения аспектов, связанных с лидерским потенциалом, мотивационными механизмами и прочими существенными для организационной действительности факторами.

\section{СПИСОК ЛИТЕРАТУРЫ}

1. Бажанов В.А. Идея нейросоциологии в современной социальной мысли // Социологические исследования. - 2017. - № 4. - C. 27-31.

2. Kalkoff W., Thye S.R., Pollok J. Developments in neurosociolgy // Sociology compass. - 2016. - V. 10. № 3. - P. 242-258.

3. McVeigh R. The neurosociology of Auguste Comte // Social science information. - 2020. - V. 59. - № 2. P. 329-354.

4. Turner J.H., Machalek R.S. The evolution of the human brain applications of neurosociology // New evolutionary sociology: recent and revitalized theoretical and methodological approaches. - NY: Routledge, 2018. - P. 337-364.

5. Шкурко Ю.С. Биосоциология и стандартная модель социальных наук: в поисках «золотой середины» // Вестник Томского государственного университета. - 2020. - № 455. - С. 91-96.

6. Шкурко Ю.С. Развитие эволюционной нейросоциологии; поведенческие биограммы и биологическая предрасположенность к социальному неравенству // Социологические исследования. - 2018. № 9. - С. 23-29.

7. Шкурко Ю.С. Нейротехнологии и пролиферация идей нейронауки // Социальная психология и общество. - 2017. - Т. 8. - № 4. - С. 32-42.

8. Сущин М.А. Масштабная нейронаука и большие науки данные // Социальные и гуманитарные науки. Отечественная и зарубежная литература. Серия 8. Науковедение: Реферативный журнал. 2019. - № 4. - C. 35-43.

9. The Brain Dialogue. URL: https://www.cibf.edu.au/discovery (дата обращения 03.02.2021).

10. Шкурко Ю.С. Инкорпорирование идей нейронауки в социологию: как преодолеть разрыв между «биологическим» и «социальным»? // Журнал социологии и социальной антропологии. - 2017. № 20 (2). - С. 22-39.

11. Шкурко Ю.С. В поисках нейросоциологии // Социологические исследования. - 2017. - № 8 (401). C. 4-11.

12. Паламарчук О.Т. Социальная нейронаука - новое фундаментальное направление большой науки // Экономика. Право. Печать. Вестник КСЭИ. - 2018. - № 2 (78). - С. 73-74.

13. Шкурко Ю.С. Следует ли социологии использовать данные биологических наук? // Социология. 2019. - № 1. - C. 101-107.

14. Rock D. Managing with the brain in mind // Strategy+business. - 2009. - № 56. URL: https://www.strategy-business.com/article/09306?gko=9efb2 (дата обращения 12.12.2020).

15. Шибкова Д.3., Байгужин П.А. Нейронаука: междисциплинарная интеграция или экспансия? // Психология. Психофизиология. - 2020. - Т. 13. - № 3. - С. 111-121.

16. Шкурко Ю.С. Нейробиология и социология: пройти между Сциллой и Харибдой. Бёртон Р. Разум vs мозг: Разговор на разных языках: пер. с англ. Ю.В. Рябининой. М.: Изд-во «Э», 2016 // Социологический журнал. - 2017. - Т. 23. - № 2. - С. 176-183.

17. Степичева А.Б., Бабанова Ю.В., Долинская А.Ю. Успех компании: эффективный лидер или умение управлять эмоциями сотрудников // Вестник ПНИПУ. Социально-экономические науки. - 2016. № 4. - C. 299-305.

18. Balconi M., Fronda G. Morality and management: an oxymoron? fNIRS and neuromanagement perspective explain us why things are not like this // Cognitive, Affective and Behavioral Neuroscience. - 2020. № 20. - P. 1336-1348 
19. Neuromanagement: the scientific approach to contemporary management / A.M. Teacu, A. Mirela, A. Capatina, D.J. Varon, P.F. Bennet, A.M. Recuerda // Proceeding of the international conference on business excellence. - 2020. - V. 14. - № 1. - P. 1046-1056.

20. Волкова А.А, Метелкина У.П., Апенько С.Н. Project Management Trends // Нейро прожект менеджмент: Материалы международной научно-практической конференции. - М.: РУСАЙНС, 2020. C. 32-36.

21. Ashkanasy N.M., Becker W.J., Waldman D.A. Neuroscience and organizational behavior: Avoiding both neuro-euphoria and neuro-phobia // Journal of Organizational Behavior. - 2014. - V. 35. - P. 909-919.

22. Waldman D.A., Balthazard P.A., Peterson S.J. Leadership and neuroscience: can we revolutionize the way that inspirational leaders are identified and developed? // Academy of Management Perspectives. - 2011. V. 25. - P. 60-74.

Поступила 12.03.2021 г. 
UDC 316.02

\title{
NEUROSCIENTIFIC INSTRUMENTS IN A MODERN SOCIAL LEARNING
}

\author{
Alina V. Smirnova, \\ sazalina@yandex.ru \\ Financial University under the Government of the Russian Federation, \\ 49, Leningradsky avenue, Moscow, 125993, Russia
}

\begin{abstract}
Alina V. Smirnova, postgraduate student, Financial University under the Government of the Russian Federation.

This article presents an analysis of using a relatively new scientific phenomenon - neuroscientific methodology - in a range of scientific disciplines (for instance, neuroeconomics, cultural neuroscience, neuropedagogy and others). The paper introduces the examples of projects and initiatives, taking place these days to develop scientific cognition. Special attention is paid to the prospect of using these methods in socio-behavioral aspects studies, including in the organizational frames. The approaches to the understanding of social neuroscience and neurosociology are given. The main dangers of sociologists (which resist active implementation of progressive methods in traditional science, including such phenomenon as «biophobia»), are mentioned. The paper introduces the publications, which have a purpose to study neuroscientific methods in a practical field: the innovative ideas, which could be realized in the sphere of human resources due to achievement of neuromanagement and neuroleadership, are noted. There are conclusions made by the authors of the research which was aimed to study neurophysiological stimulus of decision-taking in different circumstances. There are key objections, which usually appear relating to neuroscientific instruments, used in the frame of behavioral aspects study. A short characteristic of experiment showing brain activity processes and its correlation with practical role of different spheres leaders are mentioned. A brief overview of the materials of the scientific and practical conference held on the topic of neuroproject management - a scientific field aimed at studying organizational processes in which the team plays a special role - is given.
\end{abstract}

Key words: Neuroscience, neuromanagement, neuroleadership, organizational behavior, socio-behavioral aspects, human resources.

\section{REFERENCES}

1. Bazhanov V.A. Ideya neyrosotsiologii $\mathrm{v}$ sovremennoy sotsialnoy mysli [The idea of neurosociology in a modern world]. Sotsiologicheskie issledovaniya, 2017, no. 4, pp. 27-31.

2. Kalkoff W., Thye S.R., Pollok J. Developments in neurosociolgy. Sociology compass, 2016, vol. 10, no. 3, pp. 242-258.

3. McVeigh R. The neurosociology of Auguste Comte. Social science information, 2020, vol. 59, no. 2, pp. 329-354.

4. Turner J.H., Machalek R.S. The evolution of the human brain applications of neurosociology. New evolutionary sociology: recent and revitalized theoretical and methodological approaches. NY, Routledge, 2018. pp. 337-364.

5. Shkurko Yu.S. Biosotsiologiya i standartnaya model sotsialnykh nauk: v poiskakh «zolotoy serediny» [Biosociology and the social science standard model: in search of the golden mean]. Vestnik Tomskogo gosudarstvennogo universiteta, 2020, no. 455, pp. 91-96.

6. Shkurko Yu.S. Razvitie revolyutsionnoy neyrosotsiologii; povedencheskie biogrammy i biologicheskaya predraspolozhennost $\mathrm{k}$ sotsialnomu neravenstvu [The development of evolutionary sociology: behavioral bioprograms and biological disposition to the social inequality]. Sotsiologicheskie issledovaniya, 2018, no. 9, pp. 23-29.

7. Shkurko Yu.S. Neyrotekhnologii i proliferatsiya idey neyronauki [Neurotechnologies and proliferation of ideas of neuroscience]. Sotsialnaya psikhologiya i obshchestvo, 2017, vol. 8, no. 4, pp. 32-42. 
8. Sushchin M.A. Masshtabnaya neyronauka i bolshie nauki dannye [Large-scale neuro-science and scientific big-data]. Sotsialnye i gumanitarnye nauki. Otechestvennaya i zarubezhnaya literatura. Seriya 8. Naukovedenie: Referativny zhurnal, 2019, no. 4, pp. 35-43.

9. The Brain Dialogue. Available at: https://www.cibf.edu.au/discovery (accessed: 03 February 2021).

10. Shkurko Yu.S. Inkorporirovanie idey neyronauki v sotsiologiyu: kak preodolet razryv mezhdu «biologicheskim» $\mathrm{i}$ «sotsialnym»? [The incorporation of ideas of neuroscience to sociology]. Zhurnal sotsiologii $i$ sotsialnoy antropologii, 2017, no. 20 (2), pp. 22-39.

11. Shkurko Yu.S. V poiskakh neyrosotsiologii [In search of neuroscience]. Sotsiologicheskie issledovaniya, 2017, no. 8, pp. 4-11.

12. Palamarchuk O.T. Sotsialnaya neyronauka - novoe fundamentalnoe napravlenie bolshoy nauki. [Social neuroscience - a new fundamental approach in a big science]. Ekonomika. Pravo. Pechat. Vestnik KSEI, 2018, no. 2 (78), pp. 73-74.

13. Shkurko Yu.S. Sleduet li sotsiologii ispolzovat dannye biologicheskikh nauk? [Should we use data of biological sciences in sociology?]. Sotsiologiya, 2019, no. 1, pp. 101-107.

14. Rock D. Managing with the brain in mind. Strategy+business, 2009, no. 56. Available at: https://www.strategy-business.com/article/09306?gko=9efb2 (accessed: 12 December 2020).

15. Shibkova D.Z., Bayguzhin P.A. Neyronauka: mezhdistsiplinarnaya integratsiya ili ekspansiya? [Neuroscience: interdisciplinary integration or expansion?]. Psikhologiya. Psikhofiziologiya, 2020, vol. 13, no. 3, pp. 111-121.

16. Shkurko Yu.S. Neyrobiologiya i sotsiologiya: proyti mezhdu Stsilloy i Kharibdoy. Borton R. Razum vs mozg: Razgovor na raznykh yazykakh: per. s angl Yu.V. Ryabininoy. M.: Izd-vo "E”, 2016 [Neurobiology and Sociology: Walk Between Scylla and Charybdis. Burton R. Mind vs Brain: Conversation in Different Languages: translated from English Yu.V. Ryabinina. Moscow, Publishing house "E", 2016]. Sotsiologicheskiy zhurnal, 2017, vol. 23, no. 2, pp. 176-183.

17. Stepicheva A.B., Babanova Yu.V., Dolinskaya A.Yu. Uspekh kompanii: effektivny lider ili umenie upravlyat emotsiyami sotrudnikov [The company's success: effective leader or an ability to control personnel's emotions]. Vestnik PNIPU. Sotsialno-ekonomicheskie nauki, 2016, no. 4, pp. 299-305.

18. Balconi M., Fronda G. Morality and management: an oxymoron? fNIRS and neuromanagement perspective explain us why things are not like this. Cognitive, Affective and Behavioral Neuroscience, 2020, no. 20, pp. 1336-1348.

19. Teacu A.M., Mirela A., Capatina A., Varon D.J., Bennet P.F., Recuerda A.M. Neuromanagement: the scientific approach to contemporary management. Proceeding of the international conference on business excellence, 2020, vol. 14, no. 1, pp. 1046-1056.

20. Volkova A.A., Metelkina U.P., Apenko S.N. Project Management Trends. Materialy mezhdunarodnoy nauchno-prakticheskoy konferentsii "Neyro prozhekt menedzhment" [Neuro project management: Materials of the international scientific-practical conference]. Moscow, RUSAYNS Publ., 2020. pp. 32-36.

21. Ashkanasy N.M., Becker W.J., Waldman D.A. Neuroscience and organizational behavior: Avoiding both neuro-euphoria and neuro-phobia. Journal of Organizational Behavior, 2014, vol. 35, pp. 909-919.

22. Waldman D.A., Balthazard P.A., Peterson S.J. Leadership and neuroscience: can we revolutionize the way that inspirational leaders are identified and developed? Academy of Management Perspectives, 2011, vol. 25 , pp. $60-74$.

23. Kalkoff W., Thye S.R., Pollok J. Developments in neurosociolgy. Sociology compass, 2016, vol. 10, no. 3, pp. 242-258.

Received: 12 March 2021. 